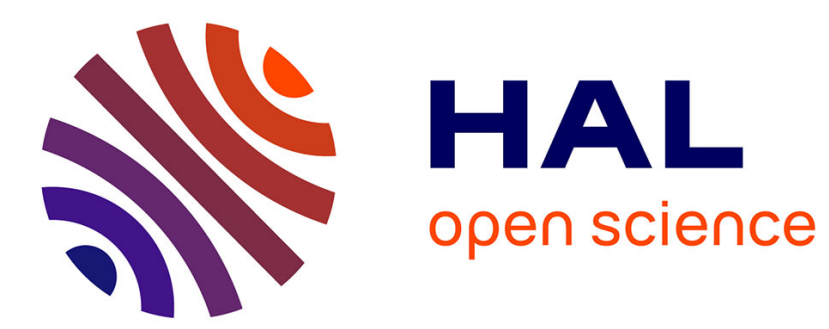

\title{
Topology Design for Integrating and Sequencing Flows in Terminal Maneuvering Area
}

Hidenori Chida, Catya Zuniga, Daniel Delahaye

\section{To cite this version:}

Hidenori Chida, Catya Zuniga, Daniel Delahaye. Topology Design for Integrating and Sequencing Flows in Terminal Maneuvering Area . Proceedings of the Institution of Mechanical Engineers, Part G: Journal of Aerospace Engineering, 2016. hal-01270717

\section{HAL Id: hal-01270717 https://hal-enac.archives-ouvertes.fr/hal-01270717}

Submitted on 22 Mar 2016

HAL is a multi-disciplinary open access archive for the deposit and dissemination of scientific research documents, whether they are published or not. The documents may come from teaching and research institutions in France or abroad, or from public or private research centers.
L'archive ouverte pluridisciplinaire HAL, est destinée au dépôt et à la diffusion de documents scientifiques de niveau recherche, publiés ou non, émanant des établissements d'enseignement et de recherche français ou étrangers, des laboratoires publics ou privés. 


\title{
Topology design for Integrating and Sequencing Flows in Terminal Maneuvering Area
}

\author{
Hidenori Chida ENAC 7, Ave. Ed Belin 31055 Toulouse, France (hidenori.chida@gmail.com) \\ Catya A. Zuñiga ENAC 7, Ave. Ed Belin 31055 Toulouse, France (catya.zuniga@ recherche.enac.fr) \\ Daniel Delahaye ENAC 7, Ave. Ed Belin 31055 Toulouse, France (delahaye@recherche.enac.fr)
}

\begin{abstract}
The current overloaded airspace near large airports leads the stakeholders to develop more fundamental means to improve the use of available air capacity in dense traffic areas as Terminal Maneuvering Area (TMA). A smart planning of aircraft trajectories and an appropriate designing of route structure can be a solution. Therefore, to analyse the capability of them, this paper proposes a novel approach to deal with the merging and sequencing problem of arrival traffic in Terminal Maneuvering Area, and examines the performance of diverse conflict resolution strategies and different TMA route structure.

A evolutionary algorithm is employed to resolve potential conflicts, altering one or more of the following decision variables; routes, speeds, and entrance time to Terminal Maneuvering Area (slot). Two different fitness functions were developed. One aims to minimize potential conflicts and the other aims to minimize at the same time potential conflicts and delay induced at runway. The performance of different combinations of decision variables, or conflict resolution strategies, is evaluated through a set of arrival aircraft to Grand Canaria airport. Also, four different route structures are tested by using the same set of incoming aircraft. The results showed that the slot and speed change and mix of all change stratagies can successfully resolve all conflicts in an efficient manner. The results also suggested that topologies with one main merging point are more efficient than the ones that have two merging points. Finally, a significant abatement of delay was observed when minimizing conflicts and delay while achieving conflict free solutions.
\end{abstract}

\section{INTRODUCTION}

To ensure the safety and fluidity of Air Transport over the twenty years, it has been necessary to revisit some key aspects through diverse modernization projects such as The Single European Sky ATM Research (SESAR) launched by the European Community and the Next Generation Air Transportation System (NextGen) launched by the US government, among others. One of the bases of these projects is to exploit technological advances in diverse fields as Communication, Navigation and Surveillance, among others, to improve the overall Air Traffic Management System.
There are a variety of airspace concepts under development to satisfy their strategic objectives such as improving safety and capacity by reducing congestion and environmental impact while increasing operational and flight efficiency. According to [12], some of the innovative Airspace Concepts to enhance capacity include a practical and efficient. organization of the airspace and its operations. Practical organization of the airspace includes among others revisiting the ATS route structure, separation minima, route spacing and obstacle clearance.

The concept of an advance Terminal Airspace Area and its operations has been introduced as part of both modernization projects due to the complex operations that are handle in it and which are facing an increasing Air Traffic demand. Every day operations are subjected to delays, frequent large turns, incompletely specified flight plans, incomplete or undefined Arrival and Departure routes, or lack of navigation systems for guidance, among others factors whose affect the performance of the overall system.

A prevalent initiative is to implement Area Navigation (RNAV) procedures as RNP Standard Terminal Arrival (STAR) and Standard Instrument Departure (SID) in the Terminal Airspace to organize incoming and outcoming flows in a more efficient manner. Procedures such as the RNAV allows to fly on any desired path without the need to fly directly toward or away from a ground-based navigation aid (NAVAID). In this case, the position of an aircraft is defined by a mix of instruments contained in the aircraft such as an inertial reference system, and the global positioning satellite system (GPS). Nowadays, more and more RNAV procedures have been implemented in both, Terminal Area (or Terminal Manoeuvre Area, TMA) and En-Route navigation. Advanced RNAV include the ability to access navigation database (that can be loaded into an aircraft Flight Management System (FMS)) to fly a path using the flight director or autopilot.

There are several potential benefits with the introduction of RNAV arrival and departure procedures, [24] including:

1) Reduce the need to vector aircraft

2) Fewer radio transmissions due to less need for controller instructions

3) Improve situational awareness

4) Reduce flying time and distance, i.e. more direct routing 
5) Reduce time and fuel consumption

6) Increased airspace/runway capacity through the use of defined paths

7) Increased flight path predictability and repeatability

8) Facilitate closely-spaced parallel arrivals and departures

A key to obtain these advantages (particularly in Terminal Airspace) is the need to design efficient arrival and departure routes as a function of the interaction between them while ensuring obstacle clearance. It has also been stated that route topologies in the Extended Terminal Airspace play an important role in scheduling performance and that an efficient scheduling and route assignment affects important performance metrics such as runway delays, throughput, fuel efficiency, and robustness to uncertainties in operations [17], [13].

In the capacity and airspace usage planning concept, the demand for efficient traffic flows plays an essential role in addressing the efficient organization of airspace volumes, which could increase the airspace capacity if properly addressed. At the same time, the airspace control management must use these forecast and analyze the strategies to minimize the risk of potential conflicts while maximizing users' initial business interests choices as often as possible (under SESAR concepts). Therefore, the future system is migrating to more predictable arrival flows where aircraft land at a specified time. This will enable air traffic control system to issue more efficiently TMA flows by providing shorter and more safety flights using more efficient airspace which will increase airspace capacity [16].

The purpose of this work is to compare diverse STAR procedures to provide efficient means to merge multiple inbound streams of aircraft and deliver them safety spaced to the runway control. It should be notice that the random arrivals must be converted into an orderly stream while merging aircraft flows coming from different entry points, commonly done in a short time horizon (about 4560 minutes). Resolution strategies have been designed to provide shorter route length and/or time to avoid conflict and to support Continuous Descent Operations. Parallel routes have been designed to avoid having bidirectional traffic on the same route providing various route options between the destination airport.

The algorithm has been successfully applied to Gran Canaria airport (Spain) with real traffic demand samples for which conflict free flow merging is produced smoothly with optimal runway feeding. The algorithm generates new speed and routes profiles aim to remove conflicts at merging points while maintain the minimum separation between aircraft following the same route according to their wake turbulence constraint.

The reminder of this works is as follows; Section II contains some of the most important related works in the field of Airspace Design, Sequencing and Merging flow of aircraft within TMA and the Conflict Detection and Resolution problem. Section III explains in detail the strategy to formulate the problem, the topologies to be analyzed and the mathematical model. A summary of the Evolutionary Algorithms (EAs) techniques are introduced in Section IV followed by the description of the model in Section V presented in Subsection III-D is described in detail. The results obtained from a sample simulation study are presented in Section IV. Finally, conclusions and future works are discussed at the end of the paper.

\section{Previous Related Works}

An aircraft approaching to TMA typically follows a STAR providing the transition from the En-Route structure to Terminal Airspace. These, landing aircraft are organized in arrival streams, i.e. the individual paths of each aircraft are gradually merged till reaching the active landing runway. It should be noticed that all aircraft are required to maintain a safe separation distance, or separation standards which are a function of the landing velocities of the leading and following aircraft as well as the size of the aircraft involved at the final approach, among other factors [9].

Before the approach takes place, each aircraft must be assigned with a landing time and a runway number. The landing time corresponds to a time window, bounded by an earliest and a latest time of arrival. Ideally, this landing time should be the same than the one in the flight plan. Unfortunately, the system is subjected to many types of perturbations in every day operations. To avoid potential conflicts at TMA, Air Traffic Control issues a set of vectoring instructions to pilot to overcome merging and sequencing of arrival flows. Delay in the landing times are induced if an aircraft is required to either slow down, hold, or change route to avoid potential conflicts. The delay is the difference between the schedule landing time and the assigned landing time and it has a cost associated to it. The cost is not only associated to fuel burn or $\mathrm{CO}_{2}$ emission, but also to the overall efficiency of the ATS causing up stream disruptions on airport operations capacity.

To properly address TMA landing operations and procedures, a search in the literature was conducted to identify the main related concepts such as the Merging and Spacing Problem and the Airspace Design and Planning process.

The aim of the Aircraft Sequencing Problem (ASP) is to optimize the assignment of aircraft to runways while optimizing the sequence of aircraft departures and arrivals on each runway. There have been different approaches since the 70s as in [9] where it was first observed that the firstcome, first-serve policy was inefficient for the medium and long term of the ASP. As a consequence of their analysis, a decision methodology termed Constrained Position Shifting 
(CPS) was proposed to eliminate the undesirable behavior. Based on the Constrained Position Shifting method, diverse approaches have been developed to solve the ASP.

Balakrishnan et al. [1] presented an study to determine the sequence that minimizes makespam for the static case of the ASP subject to diverse operational constraints. In further researches of the authors [2] and [8], an extension their work considers as a core problem maximizing runway throughput (equivalent to minimizing the makespam or the landing time of the last of a given set of aircraft) for arrivals operations, departures operations and mixed operations (simultaneous arrival and departure scheduling on a single runway). It is claimed that the output of the algorithm is not a single schedule, but a tradeoff between the likelihood of controller intervention and the time to complete runway operations for the given set of aircraft (makespam of the sequence).

In [3], the authors uses a mixed-integer zero-one formulation of the ASP together with a population heuristic algorithm. Some years later, Beasley et al. developed several studies that apply population based meta-heuristics for the formulation of the ALP using real data to validate their results, [5], [4]. They state that their work could improved Air Traffic Control decisions between $2 \%$ and $5 \%$ in terms of reducing the timespan required to land.

Dear et al. [10], [11] presented a CPS heuristic for the static and dynamic case of the Aircraft Landing Problem which aims to decide a landing time for each aircraft such that each one lands within a predetermined time window and that separation standards are respected. Other approaches solve the ASP by meta heuristics as the branch and bound algorithm as in the work of Psaraftis [22], where a dynamic programming approach of a single machine scheduling problem was developed and applied in the context of sequencing aircraft arrival operations.

A broader suite of concepts have been investigated to address merging and spacing problems arising from structured RNAV and Required Navigation Performance (RNP) routes in the Terminal environment referred as Spacing of Performance-based Arrivals on Converging Routes (SPACR) [6]. Their work addressed the near-term merging and spacing problem.

The method called "Point Merge technique" [7], [19], [15], [13], aims to merge arrival flows of aircraft without using heading instructions. It's principle is to achieve the aircraft sequence on a point with conventional direct-to instructions, using predefined legs at iso-distance to this point for path shortening or stretching.

In another approach [25], it has been described a Discrete Event model based on Coloured Petri-Nets formalism, useful to specify an algorithm focusing on the arrival phase of flight. The causal model developed considers different alternative pre-defined turning points for each flight by

\begin{tabular}{|c|ccc|}
\hline \multirow{2}{*}{ Following aircraft } & \multicolumn{3}{|c|}{ Leading aircraft } \\
heavy & medium & light \\
\hline heavy & 4 & 3 & 3 \\
medium & 5 & 3 & 3 \\
light & 6 & 4 & 3 \\
\hline \multicolumn{4}{|c}{ TABLE I } \\
SEPARATION MINIMA [NM] ICAO DOC-4444
\end{tabular}

evaluating path shortening/path stretching of all trajectories upwards the assigned merging points in a TMA.

\section{Problem Modelling}

The work consists of testing different conflict resolution strategies and diverse TMA route structure. The following sections describe in detail the modelling approach followed by the authors.

\section{A. Conflict Detection Strategy}

The Conflict Detection (CD) phase consists of predicting potential conflicts among the set of arrivals. A conflict is detected when the separation between two aircraft is smaller than a minimum separation criteria. The separation criteria depends on many factors, such as the aerodynamic requirement, the class of the two aircraft (the leading and the following) and the density of air traffic and the airspace. As different rules exist, the International separation standards which has been established by the International Civil Aviation Organization (ICAO) on the provisions of ICAO Doc 4444 (Procedures for Air Traffic Management) have been adopted. Table I summarizes the nominal horizontal separation minima based on a wake turbulence category of each aircraft.

The order of the leading and the following aircraft is determined based on arrival times at a merging node. Figure 1 illustrates this process. First, an aircraft combination is selected and a merging node is detected which is the node ahead of the aircraft concerned and shared by the two trajectories where the aircraft will fly. Next, the estimated arrival time of each aircraft is calculated with the residual distance to the merging node and its velocity. Then, based on these estimated arrival times, the leading and the following aircraft relationship is defined, yielding the corresponding separation minima (Table I). Finally, a conflict is detected when the actual separation between aircraft is smaller than the separation minima.

\section{B. Conflict Resolution Strategies}

According to [21], conflict resolution strategies are based either on the Heading Angle Change problem (HAC), the Velocity Change (VC) problem, or a mix of both cases. The $\mathrm{HAC}$ resolution approach is a method to avoid conflicts 


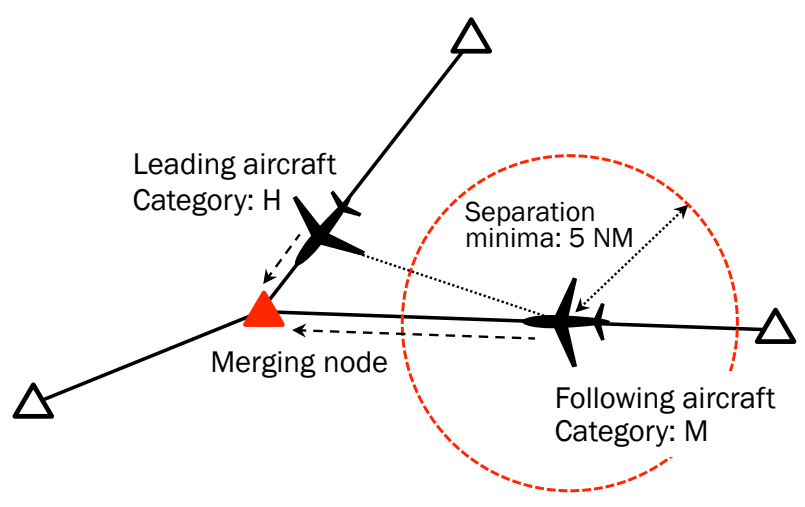

Fig. 1. Conflict detection strategy

by changing the direction of the flight while the speed remains constant during the entire manoeuvre. In the VC resolution, aircraft is allowed to change the velocity of the flight but the heading angle remains fixed. The mix of both strategy combines HAC and VC, providing an aircraft an opportunity to alter both of the heading angle and the velocity.

In addition to the route and speed change strategies, slot allocation strategy is also used in practice by Air Traffic Controllers. This strategy is also known as "holding pattern" prior to entering TMA, which allows aircraft to shift the entry time to TMA in the sequence. The goal of the slot change is to induce a delay to an aircraft so that it can avoid conflicts by switch the order of entry to TMA with another aircraft.

The resolution approaches can also be classified as cooperative or, non-cooperative. In the cooperative approach, all aircraft are assumed to coordinate their actions while in the non-cooperative approach only one of the aircraft involved in the conflict is assumed to perform the resolution manoeuvre.

The first objective of this work is to compare diverse conflict resolution strategies to evaluate the performance of each one when dealing with a set of arrivals at TMA. Therefore, based on the existing strategies, we adopt HAC, $\mathrm{VC}$, slot allocation changes and the mix of these strategies in a cooperative approach.

The HAC strategy is used as a strategic measure which determines alternative routes, or sub-routes used to avoid potential conflicts which will minimize the used of vectoring aircraft. Because this strategy is a selection of routes rather than a change of the heading angle, it is called route change strategy hereafter. The route structures, or the topology designs are briefly described in Section III-C and VI-B.

The VC strategy is translated into speed changes performed when entering TMA. Aircraft are assumed to fly

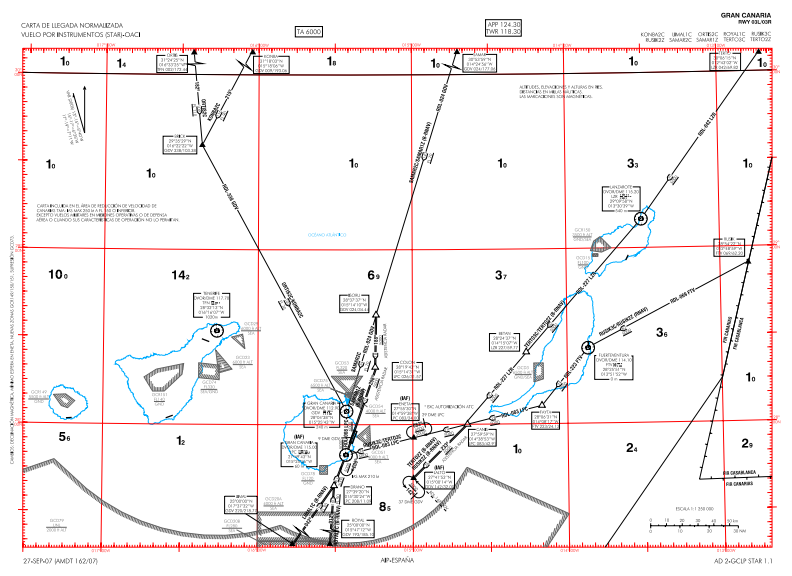

Fig. 2. Gran Canaria STAR

highest/lowest permissible speeds over each route (this value is normally considered around the $\pm 20 \%$ of its average Ground Speed). The modelling is based on the assumption of wind absence,i.e. the True Air Speed (TAS) can be considered as Ground Speed (GS). If speed resolution strategy is active, the speed is adjusted are performed at entry point and is assumed not to change the during the flight.

Some other assumptions and simplifications are made in the modelling and execution of the experiment. Aircraft trajectories were entirely deterministic. Uncertainties in the estimation of aircraft position or unexpected wind fields are not considered. Aircraft execute their planned trajectories without neither data-link transmissions, nor pilot-action delays. Conflicts among aircraft can be predicted with perfect accuracy over any time horizon. Arrival traffic management initiatives such as miles in trail restrictions, time-based metering, or ground-delay programs are not taken into account.

\section{Proposed STARs}

As for the route change strategy, different route structure designs have been analyzed to evaluate its operational performance according to some key performance indicators such as: speed profile, distance flown \& delay at runway. Therefore, as depicted in Figure 3, four distinctive TMA configurations, or topologies are proposed in this work, which have been use to performed test in the second case study.

The STAR configuration of the Gran Canaria TMA, shown in Figure 2 has been used as the reference topology layout in this work. One can see that in the arrival phase, two routes fuse into one route towards the final approach (runway 03L/03R) with merging at a single waypoint. The two arrival routes correspond to STAR defined in 
the Spanish Aeronautical Information Publication (AIP) and are called TERTO3C and RUSIK3C, starting from the entry point TERTO (30 06 ' $15^{\prime \prime} \mathrm{N}, 012$ 43'02' $\left.\mathrm{W}\right)$ and RUSIK (28 54'22'N,012 48'59'W), respectively. In all proposed topologies, three entry points are defined; two of them correspond to the STAR on Figure 2 while the third route corresponds to an additional waypoint defined so as to smoothly merge the traffic flow coming. The new route has been named as NPWT3C and starts by the entry point NPWT $\left(27^{\circ} 40^{\prime} 00^{\prime \prime} \mathrm{N}, 013^{\circ} 30^{\prime} 00^{\prime \prime} \mathrm{W}\right)$ and ends at runway.

For the design of these routes, diverse Airspace Restrictions have been taken into account as to avoid Danger, Restricted and Prohibited Areas, or constraint regarding Flight level and lateral routes dimensions. The routes from different directions towards the final approach are designed to be safety separated laterally in accordance with ICAO recommendations.

The proposed topologies consist of a route network in which aircraft are allowed to fly in order to avoid potential conflicts. Each original route has a defined number of alternative routes, or sub-routes which depend on the original route structure. One route is comprised of diverse numbers of links which join an entry point to runway. A link is defined as a portion of a route which connects two waypoints or nodes. For example, in Topology A1 depicted in Figure 3, the original route TERTO3C is represented by a plain line that begins at entry point TERTO (or Node 1) and merges with the other routes at CANIS (or Node 4). This route is conformed by an ordered list of Links: 1, 2, 3, 4, 5, 6 and 7; which are connected by Nodes: 1, 7, 8, $9,10,4,5$ and 6.5 sub-routes are attached to this original route as illustrated by dashed lines. For instance, one of these sub-routes is consisted of Link 8, 13, 14, 15, 16, 17, 18, 6 and 7. This topology design has one main merging point (Node 4), 3 original routes with 5, 8 and 4 sub-routes, making a total of 16 sub-routes.

Four topologies have distinctive features in their subroute structures and the number of merging points. For example, while topologies having letter "A" have sub-route structure like fish bones which allow aircraft to deviate from the original path, those with "B" are provided with trapezoid shaped sub-route structures. The topologies with the number "1" have only one merging point, whereas those with the number " 2 " have two merging points.

\section{Mathematical Modelling}

Consider a set of flights $\mathscr{F}=\{1, \ldots, F\}$ planned to land in a given time horizon, $[0, T]$. This time horizon is discretized by a time increment $\Delta t$, yielding a set of $\mathscr{T}=\{s * \Delta t \mid s \in \mathbb{Z}, 0 \leq s \leq T / \Delta t\}$.

Let $\mathscr{R}_{k}$ be the set of routes which start at entry point (or node) $k(k=1,2,3)$. Thus, $\mathscr{R}_{k}=\left\{r_{i k} \mid i \in \mathbb{N}, 1 \leq\right.$ $\left.i \leq R_{k}\right\}$, where $r_{i k}$ is the one route starting point $k$ and $R_{k}$ is the number of routes (main route and its attached sub-routes) associated with an entry point $k$.

Each flight $f$ in $\mathscr{F}$ has the following predetermined information:

- $e_{f}$ : TMA entry point of flight $f . e_{f} \in\{1,2,3\}$.

- $r_{f}^{s c h}$ : Scheduled route of flight $f$.

- $v_{f}^{s c h}$ : Scheduled velocity of flight $f$ at TMA entry point.

- $t_{f}^{s c h}:$ Scheduled time slot of arrival of flight $f$ at TMA entry point.

- $w t_{f}$ : Wake turbulence category of flight $f$ (heavy, medium, light).

Let $d_{f f^{\prime}}(t)$ be a distance between the leading $f$ and the following $f^{\prime}$ aircraft at time $t \in \mathscr{T}$. A conflict is detected in the following case:

$$
d_{f f^{\prime}}(t) \leq s_{f f^{\prime}} \quad \forall f, f^{\prime} \in \mathscr{F}, \forall t \in \mathscr{T},
$$

where $s_{f f^{\prime}}$ is the separation minima which depends on the aircraft's wake turbulence category of each aircraft, $w t_{f}$ and $w t_{f^{\prime}}$.

The accumulative number of conflicts $n_{f^{\prime}}$ associated with the following aircraft $f^{\prime}$ throughout the time horizon is:

$$
\begin{aligned}
n_{f^{\prime}} & =\sum_{t=0}^{T} \sum_{f=1}^{F} c_{f f^{\prime}}(t), \\
c_{f f^{\prime}}(t) & = \begin{cases}1 & \text { if } d_{f f^{\prime}}(t) \leq s_{f f^{\prime}}, \\
0 & \text { otherwise. }\end{cases}
\end{aligned}
$$

where $c_{f f^{\prime}}$ identifies a conflict in aircraft $f^{\prime}$ at instant $t$.

As introduced in Section III-B, the conflict resolution strategies involves the following decision variables: route, speed and slot regulation. These decision variables are subject to the following constraints:

\section{- Route change}

Route selection can be performed, as introduced in Section III-C, among all possible routes starting from the corresponding entry point $e_{f}$. Thus,

$$
r_{f} \in \mathscr{R}_{e_{f}}, \forall f \in \mathscr{F} .
$$

where $r_{f}$ is the route decision variable of flight $f$ after the conflict resolution strategy is applied.

\section{- Speed change}

As stipulated in Section III-B, speed can be changed within a maximum and a minimum value, being discretized in $\Delta v$ increments. Thus,

$$
v_{f} \in \mathscr{V}, \forall f \in \mathscr{F},
$$

where $\mathscr{V}=\left\{v_{\text {min }}+s * \Delta v \mid s \in \mathbb{Z}, 0 \leq s \leq\right.$ $\left.\left(v_{\max }-v_{\min }\right) / \Delta v\right\}, v_{\min }$ and $v_{\max }$ are the minimum and 


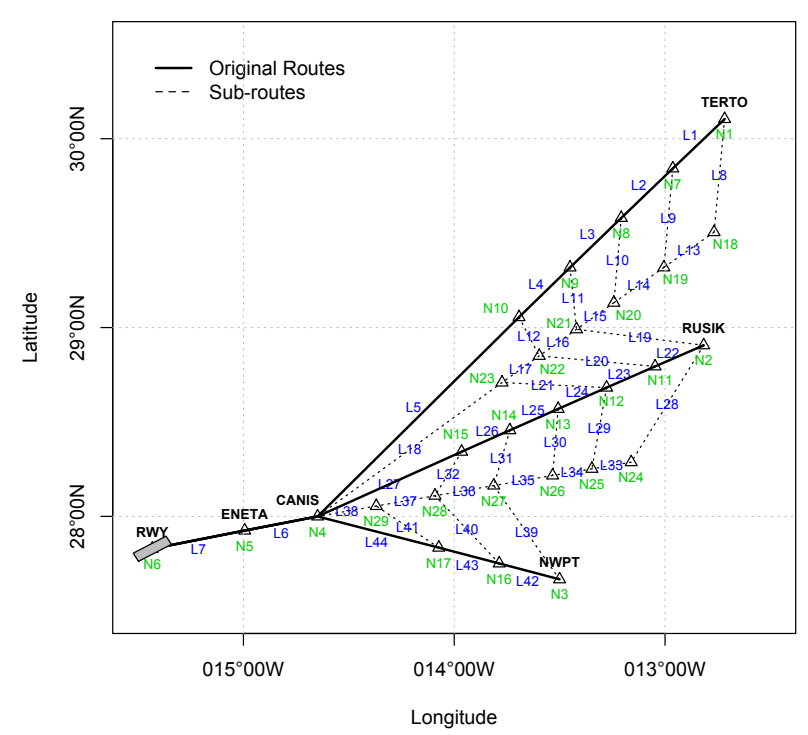

(a) Topology A1

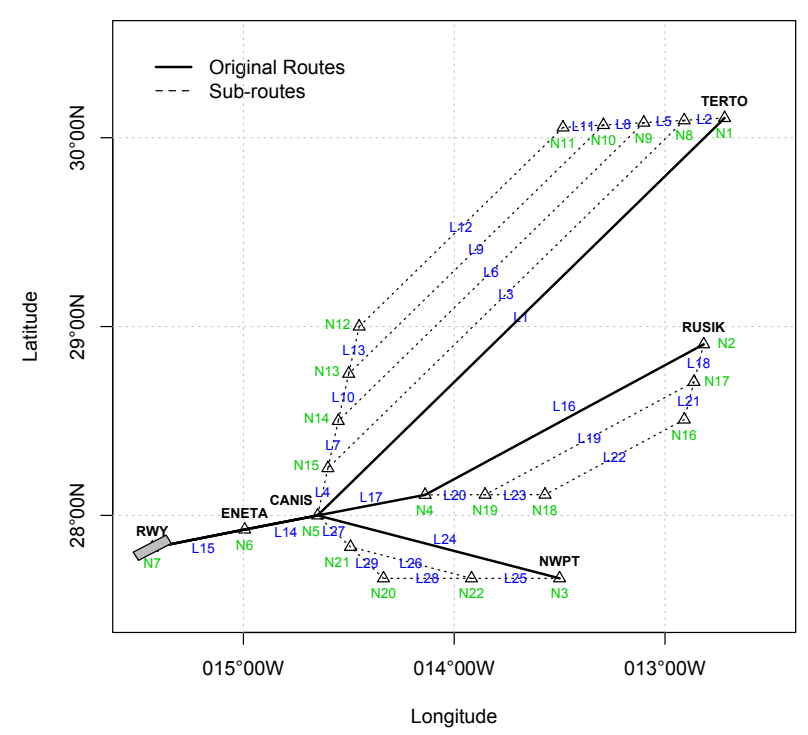

(c) Topology B1

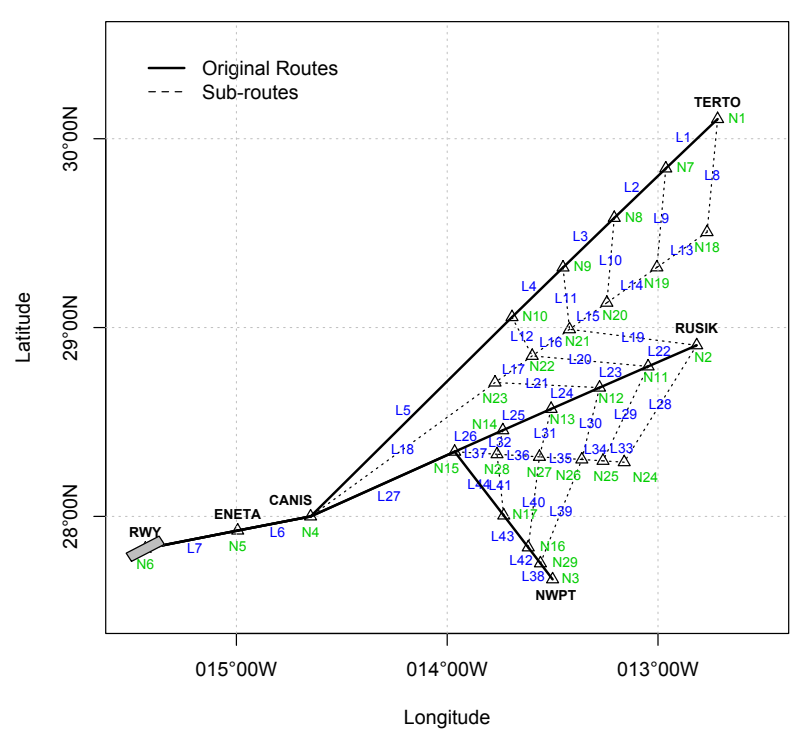

(b) Topology A2

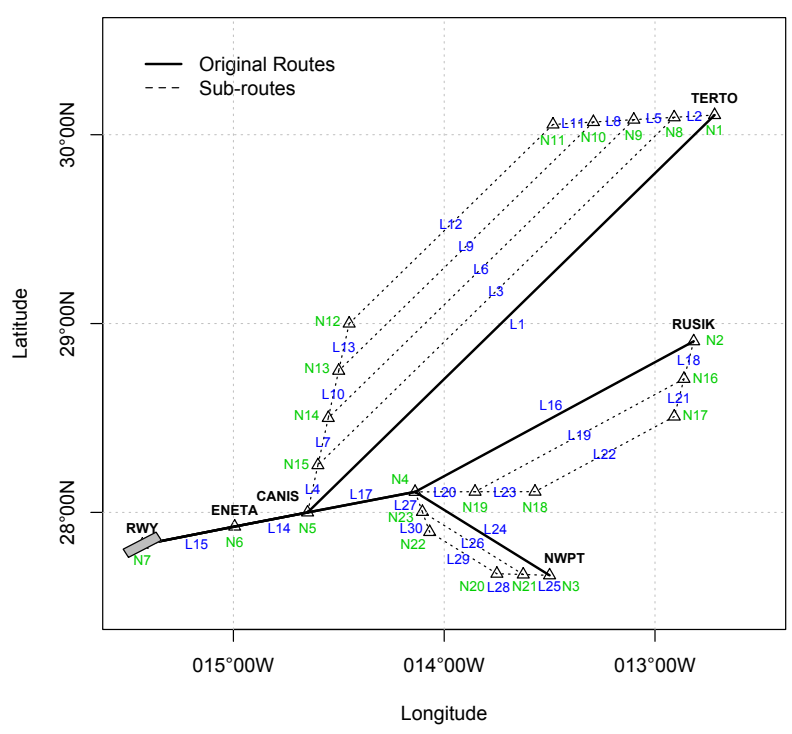

(d) Topology B2

Fig. 3. TMA configuration

maximum permissible speed values, respectively, and $v_{f}$ is the speed decision variable of flight $f$ after the conflict resolution strategy is applied.

The selected parameters for the experiment are: $\Delta v=2$ $\mathrm{kt}, v_{\min }=250 \mathrm{kt}$, and $v_{\max }=310 \mathrm{kt}$.

\section{- Slot change}

The arrival time at TMA entrance can be altered within a discretized set of allowable slot values,

$$
t_{f} \in \mathscr{T}, \forall f \in \mathscr{F},
$$

where $\mathscr{T}=\left\{t_{f}^{s c h}+s * \Delta t_{s} \mid s \in \mathbb{Z}, 0 \leq s \leq\right.$ $\left.\Delta t_{\text {smax }} / \Delta t_{s}\right\}, \Delta t_{\text {smax }}$ is the longest permissible holding time at TMA entry point, $\Delta t_{s}$ is the time increment, and $t_{f}$ is the slot decision of flight $f$ after the conflict resolution strategy is applied. Here, the selected parameters for the experiment are: $\Delta t_{s}=30$ seconds, and $\Delta t_{s \max }=300$.

Having discrete and continuous decision variables such problem belongs to the class of mix optimization problems.

Let $\mathscr{X}$ be the state space of this problem. $\mathscr{X}$ can be 
described as a set of all decisions of all flights, thus,

$$
\mathscr{X}=\left\{\left(d_{1}, \ldots, d_{F}\right) \mid d_{f} \in \mathscr{D}_{f} \text { for all } 1 \leq f \leq F\right\},
$$

where $\mathscr{D}_{f}=\left\{\left(r_{f}, v_{f}, t_{f}\right) \mid r_{f} \in \mathscr{R}_{e_{f}}, v_{f} \in \mathscr{V}, t_{f} \in \mathscr{T}\right\}$, and $d_{f}=\left\{r_{f}, v_{f}, t_{f}\right\}$ is a decision of flight $f$.

The state space dimension can be calculated by the following formula:

$$
\begin{aligned}
|\mathscr{X}| & =\prod_{f=1}^{F}\left|\mathscr{D}_{f}\right|, \\
& =\prod_{f=1}^{F} R_{e_{f}}\left(n_{\text {vmax }}+1\right)\left(n_{\text {smax }}+1\right),
\end{aligned}
$$

where $n_{v \max }$ and $n_{s \max }$ are the integer values satisfying $n_{v \max }=\left(v_{\max }-v_{\min }\right) / \Delta v$ and $n_{\operatorname{smax}}=\Delta t_{s \max } / \Delta t_{s}$ respectively.

In the proposed topology, $R_{1}=6, R_{2}=9, R_{3}=4$. When it is assumed that the average number of routes per each entry point is 6 and that $F=50,|\mathscr{X}|$ is approximately $(6 * 31 * 11)^{50}$.

\section{Evolutionary Algorithms}

Evolutionary algorithms (EAs) is an abstraction of evolutionary biology which focuses in problem solving systems based on principles of evolution and hereditary to find approximate solutions to optimization problems [14], [20], [18], [23].

The EAs maintain a population of individuals $P O P(k)=\left(x_{1}, \ldots x_{n}\right)$ for each iteration $k$. An individual represents a potential solution to the problem to be solved and is represented by a list of parameters, called chromosome or genome. In line with biological usage of the terms, it has become customary to distinguish the 'genotype' as the encoded representation of the variables, from the 'phenotype' the set of variables themselves.

To begin, several individuals are generated to form the first initial population which should be spread throughout the search space (see Figure 4). The major questions to consider are firstly the size of the population, and secondly the method by which the individuals are chosen. In respect of how the initial population is chosen, it is nearly always assumed that initialization should be 'random'; secondly, the choice of the population size must be always of a tradeoff between efficiency and effectiveness.

After the initial population is generated, it undergoes a evaluation and selection processes which identify the most adapted individual. Individuals are evaluated and assigned with a fitness score (returned by a fitness function). Based on this evaluation, individuals that have higher fitness (i.e. represent better solutions) can be identified, and these are given more opportunity to breed. The higher fitness score an individual has, higher the chances to reproduce.

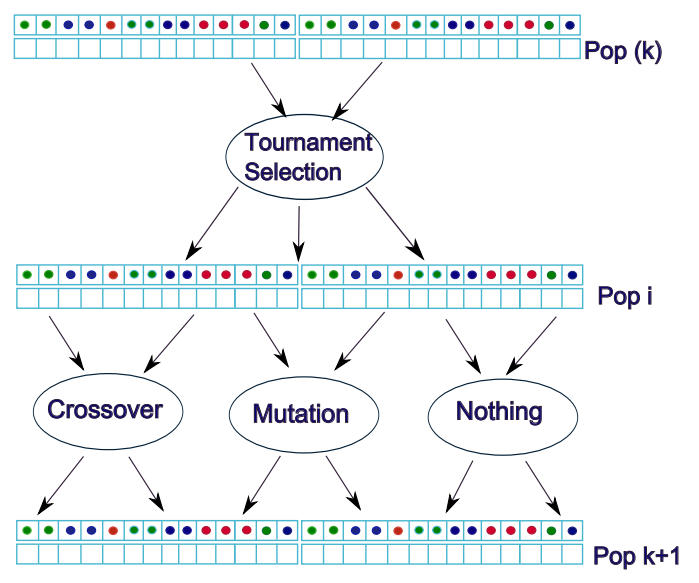

Fig. 4. Genetic Algorithm with Tournament Selection. The first step consists in the selection of the best individuals from population $P O P(k)$. Afterward, recombination operators are applied in order to produce the $P O P(k+1)$ population.

In addition, some individual of the new population undergo transformations by means of three main recombination operators to form new solutions: nothing, crossover, and mutation. The recombination of individual is carried out using simple analogies of genetic crossover and mutation.

Crossover allows solutions to exchange information (see Figure 6). One method (termed single point crossover) is to choose pairs of individuals promoted by the selection operator, randomly choose a single locus (point) within the chromosome and swap all the information to the right of this locus between the two individuals. Mutation is used to modify (flip) an individual to form another. The value of chromosomes within individual strings are then 'randomly' change. It is used very sparingly.

After recombination operators have been applied to the initial population, a new population will have been formed. This process of selection, crossover and mutation is continued until a fixed number of generations have elapsed or some form of convergence criterion has been met.

\section{Coding The EA MOdeL}

Decision variables are summarized in Fig. 5. The chromosome consists of the following 3 variables:

- Route decision (green elements)

- Speed decision (red elements)

- Slot decision (blue elements)

\section{A. Initialization}

To begin with the Evolutionary process, one must be able to initialize a population of chromosomes. 

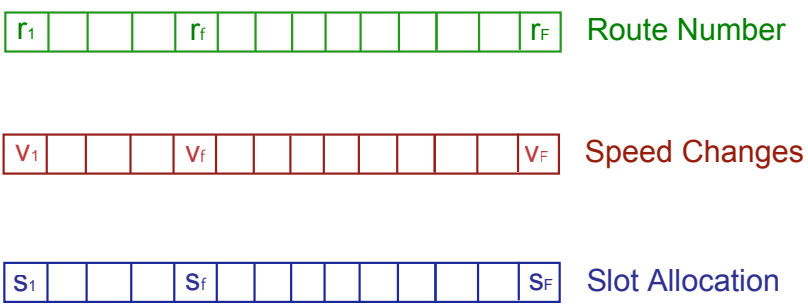

Aircraft list

Fig. 5. State Space coding

There are several methods for the initialization. One way is to use a uniform random trial to initialize the population, while one of the other possibilities is to carryout a series of initializations for each individual and then pick the highest performing values. Alternatively, estimations can be made by other methods (e.g. heuristic process) in an attempt to locate approximate solutions, and the algorithm can be started from such points. The one which has been used in our experiments is a random generator for the initial population.

\section{B. Fitness Computation}

Fitness is an evaluation score of each individual obtained by the fitness function associated with the optimization criteria. In our approach, the fitness function is associated with a number of conflicts and formulated as follows. The value of the fitness function $f_{i}\left(x_{i}\right)$ is computed as:

$$
f_{i}=\frac{1}{0.01+y_{i}} .
$$

where $y_{i}$ is the conflict ratio for each individual $x_{i} \in$ $P O P(k)$, an described as follows:

$$
y_{i}=\frac{N_{c o n}}{N_{c o n}^{0}},
$$

where, $N_{c o n}$ is a summation of conflicts of all $f$ in the individual $x_{i}$,

$$
N_{\text {con }}=\sum_{f=1}^{F} n_{f},
$$

and $N_{c o n}^{0}$ is the summation of conflicts of all $f$ when all of them fly without any decision changes, that is;

$$
\begin{aligned}
r_{f} & =r_{f}^{s c h}, \\
v_{f} & =v_{f}^{s c h}, \quad \forall f \in \mathscr{F}, \\
t_{f} & =t_{f}^{s c h} .
\end{aligned}
$$

\section{Selection}

Selection attempts to apply pressure upon the population, i.e. poorer performing individuals are weeded out and better performing, or fitter, individuals have a greater than average chance of passing the information they contain to the next generation.

The selection process which has been used in our experiments is a deterministic $(\lambda, \mu)$ tournament selection. This selection begins by randomly selecting $\lambda$ individuals from the current population, $P O P(k)$. Then, $\mu$ individuals having the highest fitness among them are selected again and added to a new population. This two steps are iterated until the generation of a new intermediate population $\mathrm{POP}_{i}(k)$ is completed.

\section{Crossover}

After the selection process, a crossover operator is applied to individuals selected with the crossover probability $p_{c}$. Crossover generates one or more offspring individuals from two parent individuals $P$ and $P^{\prime}$ which belong $P O P(k)$. The generated offsprings are added to a population of the next generation, $P O P(k+1)$. These processes ultimately result in the generation of a next population $\operatorname{POP}(k+1)$ that is different from the intermediate population (see Figure 4).

The crossover operator used in this research is bias uniform crossover, which works as the following steps (Figure 6). Firstly, two parent individuals $P$ and $P^{\prime}$ are selected randomly from the intermediate population, $P O P_{i}(k)$. Secondly, two positions of genes (flights), $i$ and $j$, are selected randomly to determine which genes to be changed. For all genes, the following probability is computed:

$$
p_{f}^{c r}=1-\frac{n_{f}}{n_{f}+n_{f}^{\prime}} \quad(i \leq f \leq j),
$$

where $n_{f}$ is the conflict number of gene (flight) $f$ of $P$, and $n_{f}^{\prime}$ is that of $P^{\prime}$.

Then, for all genes $f$ between $i$ and $j$, all decisions associated with $f$ of $P$ is copied to the gene $f$ of $P^{\prime}$ with the probability of $p_{f}^{c r}$ and those associated with $f$ of $P^{\prime}$ is copied to the gene $f$ of $P$ with the probability of $1-p_{f}^{c r}$.

When there is no conflict in both parents, $p_{f}^{c r}$ is set to $1 / 2$.

Finally, two offspring individuals different from their parents are generated, and added to the next population, $P O P(k+1)$. 


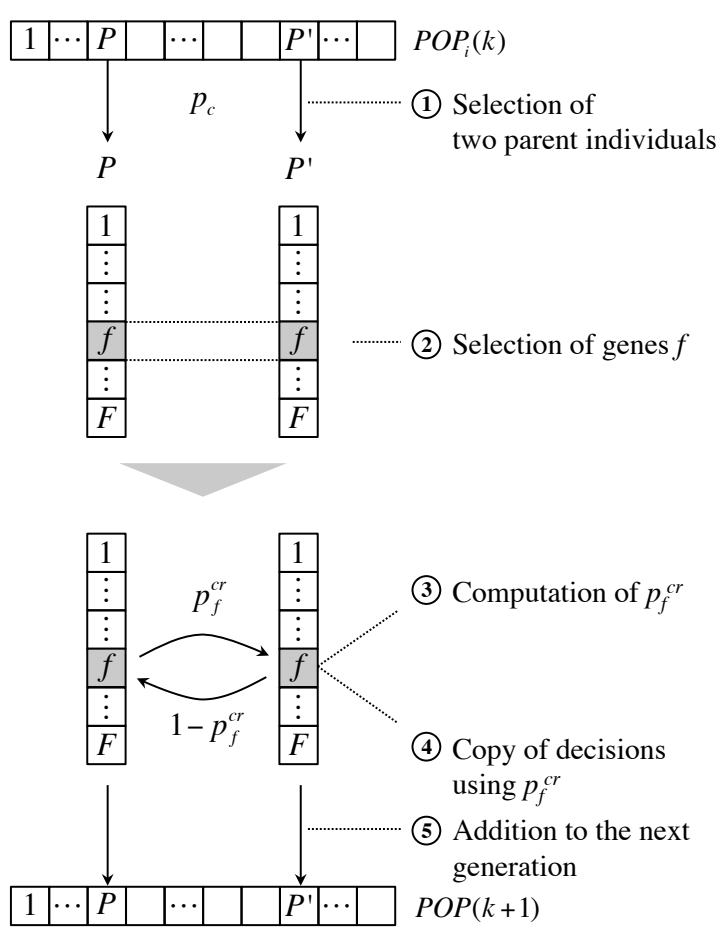

Fig. 6. Bias Crossover.

This crossover operator has the bias to transfer the decisions of the gene which has less conflicts.

\section{E. Mutation}

If an individual is not altered by the crossover, it still has a chance to be changed by the mutation operator with the probability of $p_{m} /\left(1-p_{c}\right)$, where $p_{m}$ is a mutation probability. The mutated individual is added to $\operatorname{POP}(k+$ $1)$.

In the mutation operator, it would be an issue which gene ought to be mutated and how to determined the new allele. This could be a random choice or an ordinal relation between allele values which may be more sensible to restrict the choice to alleles that are close to the current value, or bias the probability distribution. In this research, it is introduced a bias crossover operator focusing on aircraft involved in conflicts.

As shown in Figure 7, once a parent individual $P$ is selected, the bias mutation operator chooses one gene $f$ with a probability associated with the conflict number;

$$
p_{f}^{m u}=\frac{n_{f}}{N_{c o n}},
$$

and alters its decision variables.

In the selected gene, decision variables are changed randomly. For example, as for the route change, one route
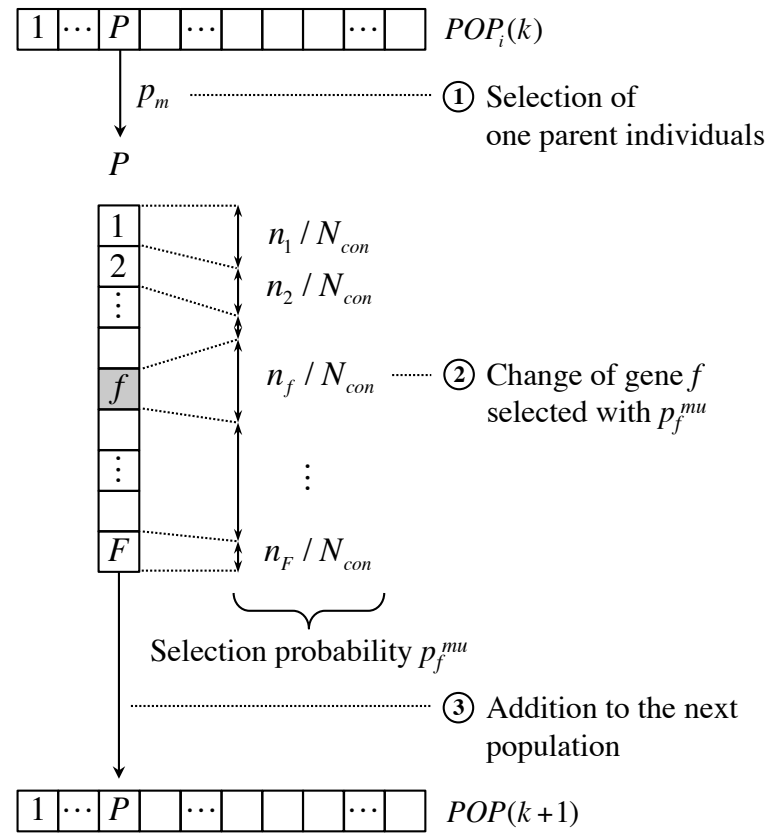

Fig. 7. Bias Mutation.

is chosen randomly from the possible set of routes $\mathscr{R}_{e_{f}}$ and $r_{f}$ is set to it.

The speed change is given by the following formula,

$$
v_{f}=v_{\min }+\Delta v * n_{v},
$$

where $n_{v}$ is a random integer variable satisfying $\left\{n_{v} \mid n_{v} \in \mathbb{Z}, 0 \leq n_{v} \leq n_{v \max }\right\}$, and $n_{v \max }=\left(v_{\max }-\right.$ $\left.v_{\text {min }}\right) / \Delta_{v}$.

The slot change is done by the following formula:

$$
t_{f}=t_{f}^{s c h}+\Delta s * n_{s},
$$

where $n_{s}$ is a random integer variable satisfying $\left\{n_{s} \mid n_{s} \in\right.$ $\left.\mathbb{Z}, 0 \leq n_{s} \leq n_{s \max }\right\}$, and $n_{\text {smax }}=\Delta s_{\max } / \Delta s$.

After the decision change, the individual is added to the next population $P O P(k+1)$.

The bias mutation operator tries to prioritize the change of gene with a larger number of conflicts.

It can be note that the objective function is non linear, not convex and not separable. The state space is not connected meaning that deterministic optimization approach are not suitable for such problem. This problem is NP-Hard. Therefore, a stochastic approach based on Evolutionary Algorithms has been developed.

\section{REsUlts}

This section has been divided in two case studies. The first one is intended to evaluate the effect of different 


\begin{tabular}{|c|c|}
\hline Parameters & Value \\
\hline Simulation runs & 30 \\
Number of generation & 500 \\
Population size & 100 \\
Probability of Crossover & 0.2 \\
Probability of Mutation & 0.8 \\
Elitism & yes \\
Selection $(\lambda, \mu)$ & $(5,3)$ \\
\hline
\end{tabular}

PARAMETERS FOR CASE STUdy I

\begin{tabular}{|c|c|c|c|}
\hline & Traffic & Heavy & Medium \\
\hline Traffic flow entering by Node 1 & $34.0 \%$ & $23.5 \%$ & $76.5 \%$ \\
Traffic flow entering by Node 2 & $38.0 \%$ & $26.3 \%$ & $73.7 \%$ \\
Traffic flow entering by Node 3 & $28.0 \%$ & $92.9 \%$ & $7.1 \%$ \\
\hline
\end{tabular}

TABLE III

CHARACTERISTICS OF THE SCENARIO WITH 50 AIRCRAFT PER HOUR

conflict resolution strategies in a cooperative approach; the second case study aims to compare diverse TMA topologies (arrival route structures) using a mix of conflict resolution strategies. Both case studies are based on one hour of arrival traffic at Gran Canaria airport. The experiments are executed on a $2.4 \mathrm{Ghz}$ Mac OS X operating system PC based on a Java code. Table II presents the parameters used to run the Evolutionary Algorithm for both case studies .

\section{A. Case Study I: Comparison of the different strategies}

The performance of each strategy: speed, route, slot change, or mix of them, is investigated in this section. Topology A1 in Figure 3 is used as the TMA design configuration for this analysis.

A traffic scenario with 50 aircraft per hour is used. Table III shows the traffic scenario in detail. It can be noticed that the most congested entry points is No. 2 corresponding to $38.0 \%$ each one of the total traffic. Within the flow entering by Node 2, the $26.3 \%$ correspond to aircraft classified as "heavy" and the remaining $73.7 \%$ is consider as "medium". The flow from Node No.1 contains $23.5 \%$ of heavy aircraft and $76.5 \%$ of medium size, composing $34.0 \%$ of total traffic flow. Finally, the remaining Node No. 3 consists of $28.0 \%$ of total flow with $92.9 \%$ of heavy aircraft and $7.1 \%$ of medium size.

Table IV summaries the major results obtained in this case study using parameter presented in Table II. For the 50 aircraft traffic scenario, 917 initial conflicts are potentially induced as shown in the row called Initial conflicts. The initial conflicts are the conflicts induced when no strategy is applied, i.e. aircraft do not use neither of speed, route or slot change.

The row called Successful runs shows the number and percentage of runs which can successfully reach the conflict free solutions. For example, having $100 \%$ in this field indicates that all runs can derive conflict free solutions while a $13.3 \%$ (as in the case of speed change strategy) indicates that only 4 out of 30 runs can find conflict free solutions. Residual conflicts field shows the number and percentage of conflicts which remained unresolved within 500 generations. The percentage is the ratio to the initial conflicts and these values are average values of 30 runs.

The other rows represent the operational performance of obtained results. These values are the average of successful runs, thus the values are NA for the route and slot change strategies, because no run can derive the conflict free trajectories.

Regarding the four strategies which use speed change (speed change alone, speed and route change combined, slot and speed change combined, and mix of all changes), it should be noted that all of the strategies have the potential to derive the conflict free solutions. While even speed change alone can reach conflict free solution in 4 runs out of 30 runs, this performance is augmented drastically when it is combined with other decision changes, being able to resolve more than $98.2 \%$ of the initial conflicts. Especially when the speed change is combined with slot change, the strategy can resolve all conflicts in all of the runs. Meanwhile, 4 strategies lead very little differences to the average and maximum modified velocity as well as the number of aircraft which are subject to velocity changes. Approximately 48 aircraft are subject to changes in its speed, resulting in flying at a new speed of $282 \mathrm{kt}$ on average.

Unlike the speed change, one can see that any of the runs with route change alone cannot reach a conflict free solution. The obtained residual conflict is the worst among 3 strategies using each change alone. However if the route change is applied in conjunction with another change or both of the others, solutions can be found in at least over 13 runs. In those strategies, the number of residual conflicts are very small ranging from $0.1 \%$ to $1.8 \%$. In the runs which are able to reach a conflict free solution, the performance on flight distance is almost identical. The average extra flight distance ranges from 5.1NM to 5.4NM and the maximum extra flight distance is 15.6NM. These manoeuvre are applied to 39.5 to 41.7 aircraft in average.

The slot change by itself also can not reach conflict free solutions, but when it is applied in conjunction with another change or the mix of all it can find solutions, as already mentioned above. As for the delay at TMA entrance induced by slot change, the route and slot change induces 90.8 seconds delay, which is longer than those in the other strategies which range from 75.2 to 78.2 seconds.

Finally, an evaluation of the average and maximum delay at runway has been done to compare all strategies. Among the strategies which can reach the conflict free solutions, the speed change alone exhibits the best performance, inducing 61.7 seconds average delay to 48.3 aircraft. This result is 


\begin{tabular}{|c|c|c|c|c|c|c|c|}
\hline Scenario & \multicolumn{7}{|c|}{50} \\
\hline fitness & \multicolumn{7}{|c|}{ Section V-B } \\
\hline Topology & \multicolumn{7}{|c|}{ A1 (Figure 3 (a)) } \\
\hline Strategy & Speed & Route & Slot & $\begin{array}{c}\text { Speed } \\
\text { +Route }\end{array}$ & $\begin{array}{l}\text { Route } \\
+ \text { Slot }\end{array}$ & $\begin{array}{l}\text { Slot }+ \\
\text { Speed }\end{array}$ & All \\
\hline Initial conflicts & \multicolumn{7}{|c|}{917} \\
\hline Residual conflict & $\begin{array}{c}38.1 \\
(4.2 \%)\end{array}$ & $\begin{array}{c}183.4 \\
(20.0 \%)\end{array}$ & $\begin{array}{c}53.0 \\
(5.8 \%)\end{array}$ & $\begin{array}{c}16.2 \\
(1.8 \%)\end{array}$ & $\begin{array}{c}4.8 \\
(0.5 \%)\end{array}$ & $\begin{array}{c}0.0 \\
(0.0 \%)\end{array}$ & $\begin{array}{c}0.1 \\
(0.0 \%)\end{array}$ \\
\hline Successful runs & $\begin{array}{c}4 \\
(13.3 \%) \\
\end{array}$ & $\begin{array}{c}0 \\
(0.0 \%)\end{array}$ & $\begin{array}{c}0 \\
(0.0 \%)\end{array}$ & $\begin{array}{c}13 \\
(43.3 \%) \\
\end{array}$ & $\begin{array}{c}15 \\
(50.0 \%)\end{array}$ & $\begin{array}{c}30 \\
(100.0 \%) \\
\end{array}$ & $\begin{array}{c}29 \\
(96.7 \%) \\
\end{array}$ \\
\hline $\begin{array}{l}\text { Average modified } \\
\text { velocity }[\mathrm{kt}]\end{array}$ & 281.7 & NA & NA & 282.6 & 0.0 & 282.1 & 283.2 \\
\hline $\begin{array}{l}\text { Maximum modified } \\
\text { velocity [kt] }\end{array}$ & 309.5 & NA & NA & 309.1 & 0.0 & 309.5 & 309.4 \\
\hline $\begin{array}{l}\text { Number of aircraft with } \\
\text { velocity changes }\end{array}$ & 48.3 & NA & NA & 48.5 & 0.0 & 48.2 & 48.1 \\
\hline $\begin{array}{l}\text { Average extra flight } \\
\text { distance }[\mathrm{nm}]\end{array}$ & 0.0 & NA & NA & 5.3 & 5.1 & 0.0 & 5.4 \\
\hline $\begin{array}{l}\text { Maximum extra flight } \\
\text { distance }[\mathrm{nm}]\end{array}$ & 0.0 & NA & NA & 15.6 & 15.6 & 0.0 & 15.6 \\
\hline $\begin{array}{l}\text { Number of aircraft with } \\
\text { route changes }\end{array}$ & 0.0 & NA & NA & 41.7 & 39.5 & 0.0 & 41.1 \\
\hline $\begin{array}{l}\text { Average delay at TMA } \\
\text { entrance }[\mathrm{sec}]\end{array}$ & 0.0 & NA & NA & 0.0 & 90.8 & 78.2 & 75.2 \\
\hline $\begin{array}{l}\text { Maximum delay at TMA } \\
\text { entrance [sec] }\end{array}$ & 0.0 & NA & NA & 0.0 & 298.0 & 297.0 & 299.0 \\
\hline $\begin{array}{l}\text { Number of aircraft with } \\
\text { slot changes }\end{array}$ & 0.0 & NA & NA & 0.0 & 29.7 & 25.4 & 24.2 \\
\hline $\begin{array}{l}\text { Average delay at runway } \\
{[\mathrm{sec}]}\end{array}$ & 61.7 & NA & NA & 128.4 & 152.3 & 140.3 & 199.7 \\
\hline $\begin{array}{l}\text { Maximum delay at } \\
\text { runway [sec] }\end{array}$ & 281.7 & NA & NA & 399.4 & 434.2 & 524.7 & 594.7 \\
\hline $\begin{array}{l}\text { Number of aircraft } \\
\text { delayed }\end{array}$ & 48.3 & NA & NA & 43.7 & 45.2 & 41.7 & 46.0 \\
\hline
\end{tabular}

RESUlts OF CASE STUDY I: COMPARISON OF STRATEGIES

followed by the speed and route change strategy, which shows 128.4 seconds average delay to 43.7 aircraft.

Figure 8 and 9 represents the evolution of average fitness and the average conflict ratio $\bar{y}=\sum_{i} y_{i} / F$ through the generations. Here, one can see that slot and speed change strategy can reach the saturation fastest, which is followed by mix of all. While fitness growth of route and slot strategy is faster than that of speed and route strategy until 200 generations, it decelerates its pace after that point and surpassed by the speed and route strategy. Eventually, the route and slot shows the worst conflict resolution performance among the strategies using two decision changes. It is also interesting to point out that while speed change alone and slot change alone still shows potentials to grow their fitness at 500 generation, route change alone already shows the saturation of fitness.

These results suggest several implications. Firstly, speed decision has the highest potential in resolving conflicts among the 3 decisions. Secondly, when more than two decision variables are combined, the efficiency in deriving solutions is largely increased, and above all slot and speed change strategy and mix of all strategy can resolve conflicts quiet effectively. Thirdly, as the number of decision variables used in EA increases, larger delay is imposed at runway due to the nature of the conflict resolution strategies.

B. Case Study II: Comparison of the different topologies with the two traffic scenarios

The previous case study is intended to test the benefits of a set of strategies to minimizes the total number of conflicts of an arrival in on hour traffic scenario. For the second case study, it is aimed to compare the efficiency of different TMA design configurations by minimizing both, the total number of conflicts, and the delay of each aircraft. Therefore, other fitness function has been develop to evaluate the benefit of the delay minimization.

Tests are conducted with two different fitness functions: the first fitness function deals with the minimization of the total number of conflicts, as described in Section V-B; while the second fitness function minimizes the delay time at the 


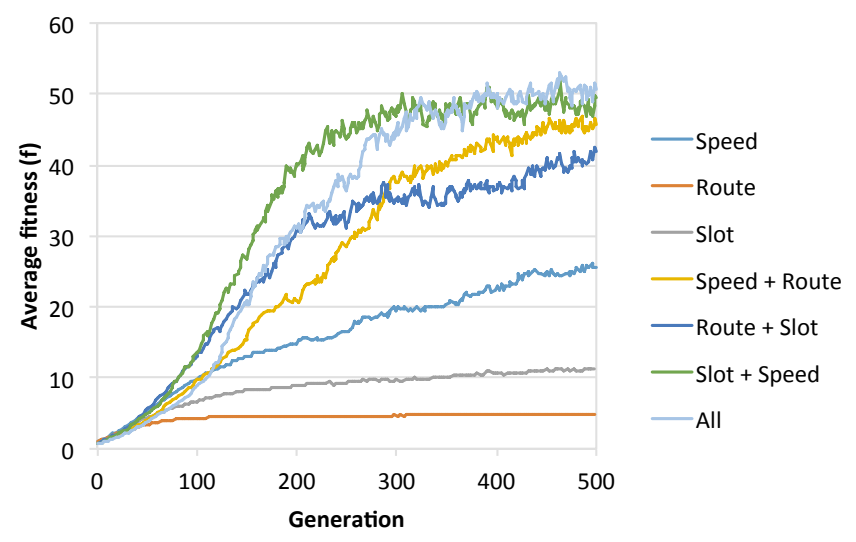

Fig. 8. Evolution of average fitness

runway, and is defined as follows:

$$
\begin{aligned}
f_{i} & =\frac{1}{0.01+y_{i}}+\frac{1}{0.01+z_{i}} \\
z_{i} & =\frac{\sum_{f=1}^{F} \max \left\{0,\left(t_{f}^{a}-t_{f}^{a s c h}\right) / 60\right\}}{F},
\end{aligned}
$$

where $t_{f}^{a s c h}$ is the scheduled arrival time at the runway of flight $f, t_{f}^{a}$ is an arrival time after the conflict resolution strategies are applied, and $z_{i}$ represents the average of delay caused by the strategies for all aircraft, normalized by 60 seconds.

The performance of diverse topologies depicted in Figure 3 where tested within this Section. The traffic scenario used is presented in Table III. The parameters used to tune the Evolutionary Algorithm are the ones presented in Table II, except for the number of generations. For this experiment, 1500 generation is adopted to see the evolution of delay more clearly.

Table V summaries the major results obtained in this case study. Different numbers of initial conflicts are predicted for each topology. For example, for Topology B1, it has been found 842 potential Initial conflicts meanwhile Topology A2 predicts 1228 potential Initial conflicts. It should be noted that for both sub-route structures (A and B), two merging points always impose higher level of initial conflicts. This is because in the two merging points cases, aircraft from entry node 2 and 3 need to be merged and are required to fly in the same link earlier than in the one merging point cases.

The Residual conflict row shows that more than $99.9 \%$ of the initial conflicts are resolved in all topologies, while the Successful runs row shows that the worst case is to use Topology A2 with minimizing only conflicts, which succeeds in resolving all conflict in 25 runs of 30.

In both fitness functions, four topologies propose similar results regarding the velocity. When minimizing only con-

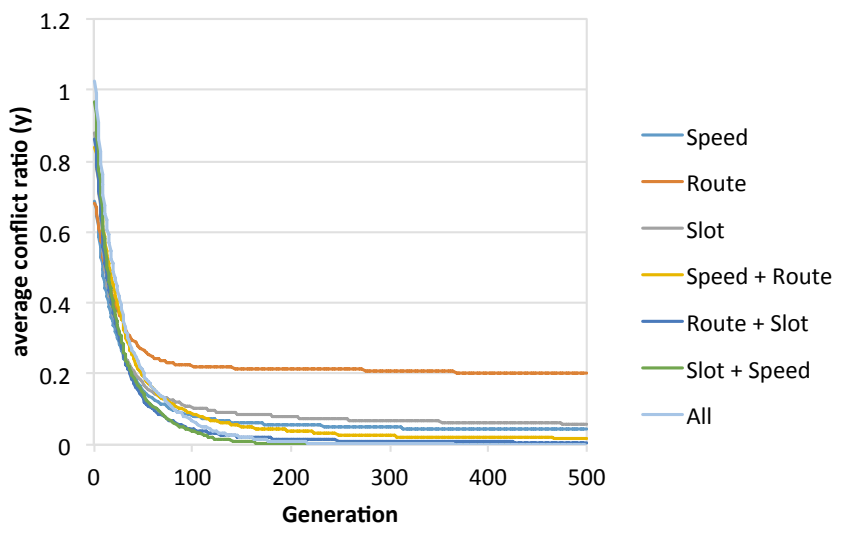

Fig. 9. Evolution of average conflict ratio $(\bar{y})$

flicts, the average modified velocity varies between 281.6 to $284.1 \mathrm{kt}$, Maximum modified velocity is approximately $309 \mathrm{kt}$, and about 48 aircraft are subject to change their velocity. When minimizing both conflicts and delay, the strategy changes the velocity of approximately 44 aircraft, which results in the Average modified velocity of $298.1 \mathrm{kt}$ to $300.3 \mathrm{kt}$ and the Maximum modified velocity of $310 \mathrm{kt}$. It should be noted that adding the delay minimization fitness increases the velocities and reduce the number of decision changes.

Unlike the velocity changes, four topologies bring some changes to the results regarding the flight distance performance in both fitness functions. For example, it is interesting to see that Topologies " $\mathrm{B}$ " is better in Number of aircraft with route changes, inducing less aircraft to change their route decisions than Topologies "A". Regarding the distance, when dealing only with the minimization of conflicts, the Average extra flight distance ranges between 4.9 $\mathrm{NM}$ to $5.9 \mathrm{NM}$. When minimizing both conflict and delay, the Average extra flight distance is reduced by between $50 \%$ and $60 \%$, ranging from $2.6 \mathrm{NM}$ to $3.5 \mathrm{NM}$. The worst efficient design in term of extra distance is A2, adding the largest extra distance and the number of decision changes.

The average delay at TMA entrance is also subject to change by the topology difference. When minimizing only conflicts, topologies having one merging point seem to be the most efficient, introducing an average delay of approximately 74 seconds to 24 aircraft. When minimizing both conflicts and delay, this feature is also the same, but between $\mathrm{A} 1$ and $\mathrm{B} 1$, Topology A1 shows better performance. As expected, one can see the abatement of delay level at TMA when minimizing both conflicts and delay.

The total Delay time at runway is taken as a key performance indicator to compare the topology efficiency. In this sense, when minimizing only conflicts the most efficient 


\begin{tabular}{|c|c|c|c|c|c|c|c|c|}
\hline \multirow{3}{*}{$\begin{array}{l}\text { Scenario } \\
\text { fitness } \\
\text { Topology } \\
\end{array}$} & \multicolumn{8}{|c|}{50} \\
\hline & \multicolumn{4}{|c|}{ conflict } & \multicolumn{4}{|c|}{ conflict + delay } \\
\hline & A1 & A2 & B1 & $\mathrm{B} 2$ & A1 & A2 & $\mathrm{B} 1$ & $\mathrm{~B} 2$ \\
\hline Initial conflicts & 917 & 1228 & 842 & 997 & 917 & 1228 & 842 & 997 \\
\hline Residual conflicts & $\begin{array}{c}0.1 \\
(100.0 \%)\end{array}$ & $\begin{array}{c}1.4 \\
(99.9 \%)\end{array}$ & $\begin{array}{c}0.0 \\
(100.0 \%)\end{array}$ & $\begin{array}{c}0.2 \\
(100.0 \%)\end{array}$ & $\begin{array}{c}0.1 \\
(100.0 \%)\end{array}$ & $\begin{array}{c}0.4 \\
(100.0 \%)\end{array}$ & $\begin{array}{c}0.0 \\
(100.0 \%)\end{array}$ & $\begin{array}{c}0.0 \\
(100.0 \%)\end{array}$ \\
\hline Successful runs & $\begin{array}{c}29 \\
(96.7 \%) \\
\end{array}$ & $\begin{array}{c}25 \\
(83.3 \%) \\
\end{array}$ & $\begin{array}{c}30 \\
(100.0 \%)\end{array}$ & $\begin{array}{c}27 \\
(90.0 \%) \\
\end{array}$ & $\begin{array}{c}29 \\
(96.7 \%) \\
\end{array}$ & $\begin{array}{c}29 \\
(96.7 \%) \\
\end{array}$ & $\begin{array}{c}30 \\
(100.0 \%)\end{array}$ & $\begin{array}{c}30 \\
(100.0 \%)\end{array}$ \\
\hline $\begin{array}{l}\text { Average modified } \\
\text { velocity }[\mathrm{kt}]\end{array}$ & 283.2 & 284.1 & 282.7 & 281.6 & 300.2 & 298.1 & 300.3 & 298.8 \\
\hline $\begin{array}{l}\text { Muximum modified } \\
\text { velocity }[\mathrm{kt}]\end{array}$ & 309.4 & 309.6 & 309.5 & 309.5 & 310 & 310 & 310 & 310 \\
\hline $\begin{array}{l}\text { Number of aircraft with } \\
\text { velocity changes }\end{array}$ & 47.9 & 48.1 & 48.6 & 48.7 & 42.8 & 44.1 & 43.1 & 43.9 \\
\hline $\begin{array}{l}\text { Average extra flight } \\
\text { distance }[\mathrm{nm}]\end{array}$ & 5.4 & 5.9 & 5.2 & 4.9 & 2.6 & 3.5 & 2.7 & 2.8 \\
\hline $\begin{array}{l}\text { Maximum extra flight } \\
\text { distance }[\mathrm{nm}]\end{array}$ & 15.6 & 14.3 & 14.4 & 14.3 & 13.6 & 13.9 & 13.2 & 13.1 \\
\hline $\begin{array}{l}\text { Number of aircraft with } \\
\text { route changes }\end{array}$ & 41.1 & 41.4 & 35.5 & 35.5 & 26.1 & 29.2 & 21.4 & 24.3 \\
\hline $\begin{array}{l}\text { Average delay at TMA } \\
\text { entrance }[\mathrm{sec}]\end{array}$ & 75.2 & 84.7 & 73.4 & 83.9 & 23.5 & 38.4 & 30.3 & 36.6 \\
\hline $\begin{array}{l}\text { Maximum delay at TMA } \\
\text { entrance }[\mathrm{sec}]\end{array}$ & 299 & 300 & 292 & 297.8 & 240 & 275.2 & 268 & 272 \\
\hline $\begin{array}{l}\text { Number of aircraft with } \\
\text { slot changes }\end{array}$ & 24.2 & 26.3 & 23.6 & 25.9 & 10.2 & 14.2 & 11.9 & 14.3 \\
\hline $\begin{array}{l}\text { Average delay at runway } \\
{[\mathrm{sec}]}\end{array}$ & 199.7 & 215.3 & 199.8 & 214 & 35.3 & 68.7 & 43.0 & 55.4 \\
\hline $\begin{array}{l}\text { Maximum delay at } \\
\text { runway }[\mathrm{sec}]\end{array}$ & 594.7 & 593.1 & 618.5 & 644.1 & 373.3 & 441.2 & 418.1 & 440.2 \\
\hline $\begin{array}{l}\text { Number of aircraft } \\
\text { delayed }\end{array}$ & 46 & 45.8 & 45.8 & 46.5 & 26 & 30.6 & 26.4 & 28.8 \\
\hline
\end{tabular}

TABLE V

RESULTS OF CASE STUDY II: COMPARISON OF THE DIFFERENT TOPOLOGIES

topologies are topologies having one merging point. When minimizing conflicts and delays, this is also the case but Topology A1 shows better results than B1, inducing a total average delay of 35.3 seconds to 26 aircrafts. Here, one can also see the significant abatement of the Delay time at runway and the number of delayed aircraft by using delay optimization.

Figure 10 and Figure 11 depict the evolution of the average fitness and the average conflict ratio $(\bar{y})$ respectively. As depicted in Figure 11, conflict ratio decreases almost to zero after 200 or 300 generations in all topologies. Figure 10 reveals that Topology A2 has the worst performance in fitness evolution while the other three topologies show almost the same performance.

Figure 12 shows the evolution of the average fitness for all topologies when minimization delay in conjunction with conflicts. Also in this case, the performance of Topologies $\mathrm{A} 1, \mathrm{~B} 1$ and $\mathrm{B} 2$ is quite similar except for Topology A2 which has lower efficiency in reaching conflict free solutions. The associated evolutions of the average conflict ratio $(\bar{y})$ and the average delay at runway $(\bar{z})$ are given in Figure 13. Here, one can see that the average conflict ratios reach to almost zero after 200 or 300 generations. After achieving this enough abatements of conflicts, the average delays at runway start to decrease. In Figure 13, it should be noted that Topology A2 imposes higher delay at runway in the early stage of generation, while the other topologies impose the same level of delay. Also it is interesting to point out that topologies having one merging point achieve faster decrease of delay at runway than the topologies having two merging points.

As overall conclusions, some points are suggested. First, the results shows that one merging point topologies have better performance especially in delay criteria. Secondly, the experiment reveals that Topology B1 is the most efficient when minimizing only conflicts because of the performance in route decision change. Thirdly, Topology A1 is the most efficient when minimizing both conflicts and delay, because of its delay performance.

\section{CONCLUSIONS}

The imminent growth of the air traffic requires a broadly applicable and reliable set of Decision Support tools to improve strategic planning in the Air Traffic Management domain. Such tools must be able to cope efficiently and safely with the growing demand. 


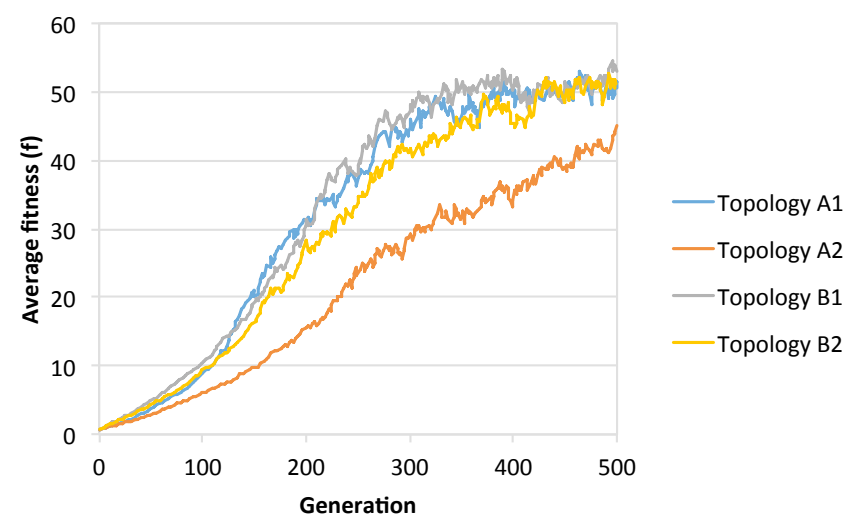

Fig. 10. Evolution of average fitness when minimizing only conflicts

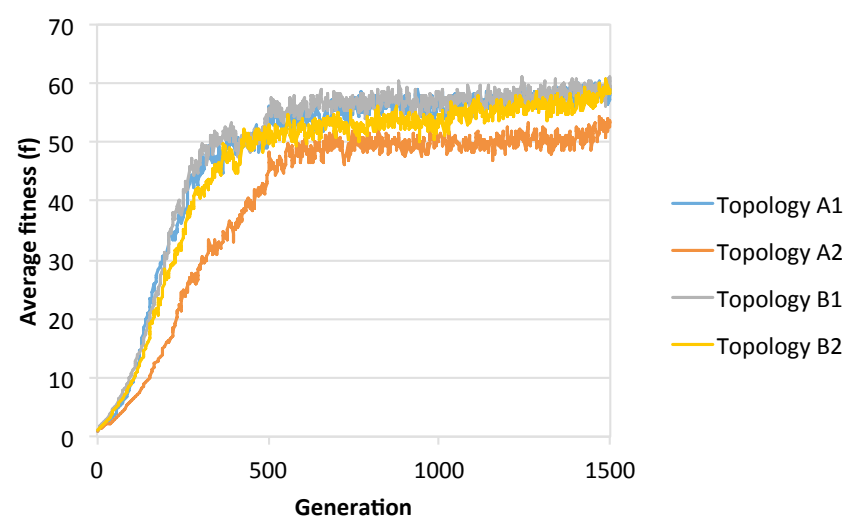

Fig. 12. Evolution of average fitness when minimizing conflicts and delay

A novel approach for the aircraft sequencing and merging problem in TMA was presented. An Evolutionary Algorithm was adopted to tackle this problem, for which chromosome coding and several computational operators have been developed. For each aircraft, route, speed \& entry time can be changed as decision variables to find a set of conflict free trajectories. This algorithm has been applied to Gran Canaria airport in Spain with real traffic demand samples.

The first objective of this work is to do a comparative study among strategies to analyzed how the different strategies affect the various performance like efficiency in conflict resolution and delay time. In this case study, the results showed that slot and speed strategy is the most effective to resolve conflicts within TMA. This strategy induces to $84 \%$ of the aircraft an average delay at runway of 140 seconds. The mix of all strategy also shows the efficient conflict resolution, but this time $92 \%$ of aircraft are delayed at runway for 199 seconds.

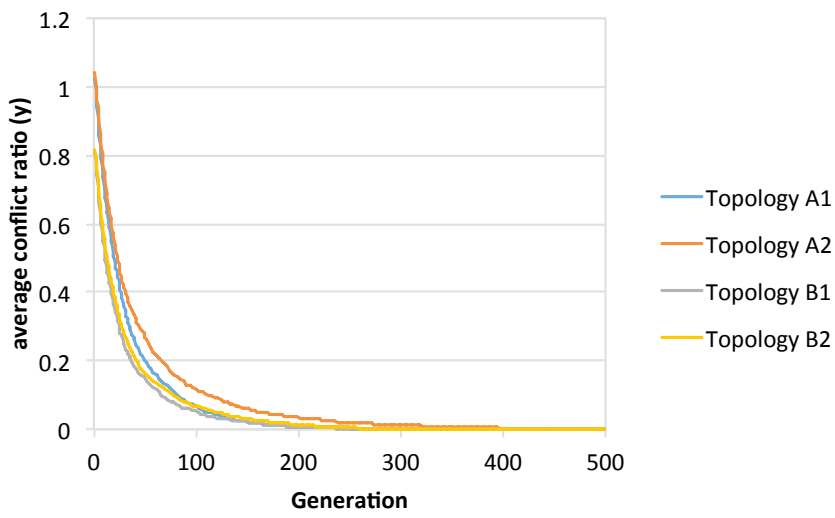

Fig. 11. Evolution of average conflict ratio $(\bar{y})$ when minimizing only conflicts

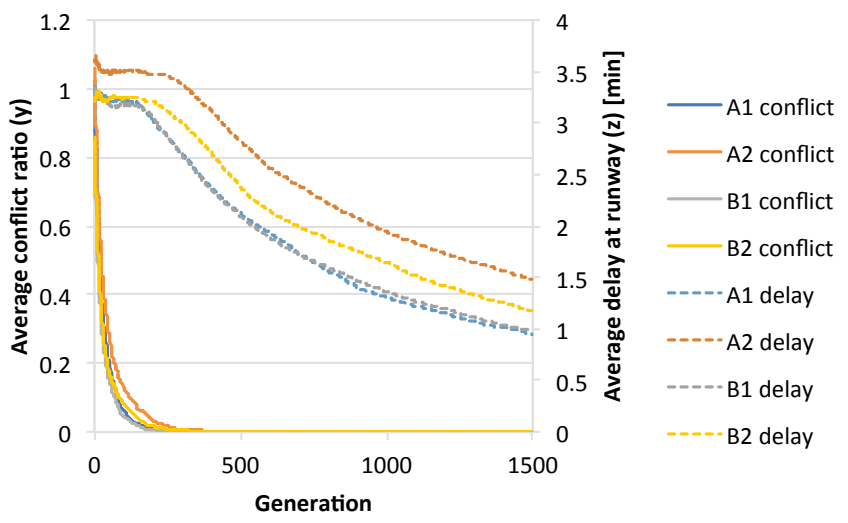

Fig. 13. Evolution of average conflict ratio $(\bar{y})$ and average delay at runway $(\bar{z})$ when minimizing conflicts and delay

The second objective of this work is to investigate the effect of different topologies designs for TMA. Therefore, four topology designs were proposed as TMA route structure. In this case study, it has been found that topologies having one merging point usually impose less delay. Also it is shown that Topology B1 is the most efficient when minimizing only conflicts, whereas Topology A1 is the most efficient when minimizing delay at runway. Through this case study, it was also demonstrated that incorporating delay evaluation in the fitness function successfully worked and the algorithm could abate the delay level significantly.

Further research can be done in order to develop a continuous model approach to deal with the route change strategy at TMAs. To this aim, the scheduling model and algorithm must be improved with, for example the Constrained Position Shifting (CPS) method [2]. Other research directions concern with the development of algorithms for computing optimal routes from En-Route sectors. The model can also be improved to test the sequencing algorithm in a dynamic 
simulation in which aircraft are added to the entry queue as soon as they arrive and being removed from the queue as they land.

\section{ACKNOWLEDGMENT}

This research effort has been partly funded by a project in the air traffic management domain with the aim of providing a significant contribution to the attainment of the common European goals set by the SESAR program and beyond. The project has been called XXX by XXX. Other part of the founding come from the Mexican Institution called Consejo Nacional de Ciencia y Tecnología (CONACyT) whose efforts have been also very important.

\section{REFERENCES}

[1] H. Balakrishnan and B. Chandran. Scheduling aircraft landings under constrained position shifting. In The AIAA Guidance, Navigation and Control Conference, Keystone, Colorado., 2006.

[2] H. Balakrishnan and Bala G. Chandran. Algorithms for scheduling runway operations under constrained position shifting. Oper. Res., 58:1650-1665, November 2010.

[3] J.E. Beasley, M. Krishnamoorthy, Y. M. Sharaiha, and D. Abramson. Scheduling aircraft landings - the static case. Transportation Science, 34:180-197, 2000.

[4] J.E. Beasley, M. Krishnamoorthy, Y. M. Sharaiha, and D. Abramson. Displacement problem and dynamically scheduling aircraft landings. Journal of the Operational Research Society, 55:5, 2004.

[5] J.E. Beasley, J. Sonander, and P. Havelock. Scheduling aircraft landings at london heathrow using a population heuristic. Journal of the Operational Research Society, 52:483-493, 2001.

[6] T.A. Becker, D.R. Barker, and A.P. Smith. Methods for maintaining benefits for merging aircraft on terminal rnav routes. In Digital Avionics Systems Conference, 2004. DASC 04. The 23rd, volume 1, pages 2.E.1 - 21-13 Vol.1, oct. 2004.

[7] L. Boursier, B. Favennec, E. Hoffman, A. Trzmiel, F. Vergne, and K. Zeghal. Integrating aircraft flows in the terminal area with no radar vectoring. In 6th AIAA Aviation Technology, Integration and Operations Conference (ATIO) 25 - 27 September 2006, Wichita, Kansas, 2006.

[8] B. Chandran and H. Balakrishnan. A dynamic programming algorithm for robust runway scheduling. In American Control Conference, 2007. ACC '07, pages $1161-1166$, july 2007.

[9] R. G. Dear. The dynamic scheduling of aircraft in the near terminal area. Technical report, MIT, 1976.

[10] Roger G. Dear and Yosef S. Sherif. The dynamic scheduling of aircraft in high density terminal areas. Microelectronics Reliability, 29(5):743 - 749, 1989.

[11] Roger G. Dear and Yosef S. Sherif. An algorithm for computer assisted sequencing and scheduling of terminal area operations. Transportation Research Part A: General, 25(2-3):129 - 139, 1991.

[12] EUROCONTROL. Airspace concept handbook for the implementation of performance based navigation (pbn). Technical report, EUROCONTROL, 2012.

[13] B. Favennec, T. Symmans, D. Houlihan, K. Vergne, and K. Zeghal. Point merge integration of arrival flows enabling extensive rnav application and cda - operational services and environment definition. Technical report, EUROCONTROL, 2008.

[14] D.E Goldberg. Genetic Algorithms in Search, Optimization and Machine Learning. Reading MA Addison Wesley, 1989.

[15] E. Hofhan, d. Ivanescu, C. Shaw, and K. Zeghal. Analysis of spacing guidance for sequencing aircraft on merging trajectorie. In 21st Digital Avionics Systems Conference, Irvine, California, October 2002, 2002.
[16] J. Hull, B. Barmore, and T. Abbott. Technology-enabled airborne spacing and merging. In Digital Avionics Systems Conference, 2004. DASC 04. The 23rd, volume 1, pages 2.B.4 - 2.1-9 Vol.1, oct. 2004.

[17] D. Ivanescu, C. Shaw, C. Tamvaclis, and T. Kettunen. Models of air traffic merging techniques: Evaluating performance of point merge. In American Institute of Aeronautics and Astronautics, 2009, September 2009.

[18] J.R Koza. Genetic Programming. MIT press, 1992.

[19] Boursier L., Favennec B., Hoffman E., Trzmiel A., Vergne F., and Zeghal K. Merging arrival flows without heading instructions. In 7th USA/Europe Air Traffic Management R\&D Seminar, 2007.

[20] Z Michalewicz. Genetic algorithms + Data Structures $=$ Evolution Programs. Springer-verlag, 1992.

[21] L. Pallottino, E. Feron, and Bicchi A. Conflict resolution problems for air traffic management systems solved with mixed integer programming. Intelligent Transportation Systems, IEEE Transactions on, Vol. 3, No. 1. (2002), pp. 3-11., 2002.

[22] H. N. Psaraftis. A dynamic programming approach for sequencing groups of identical jobs. Journal of the Operational Research Society, 28:1347-1359, Nov. - Dec. 1980.

[23] H.P Schwefel. Evolution and Optimum Seeking. Wiley, New York, 1995.

[24] S. Szurgyi, S. Shresta, D. Neskovic, J. DeArmon, and S. Williams. Analysis of observed aircraft-to-aircraft separations. In Integrated Communications, Navigation and Surveillance Conference, 2008. ICNS 2008, pages $1-11$, may 2008.

[25] C.A. Zuniga, M.A. Piera, S. Ruiz, and I. Del Pozo. A cd\&cr causal model based on path shortening/path stretching techniques. Transportation Research Part C: Emerging Technologies, pages -, 2012. 